\title{
The 2015 Albert Lasker Basic Medical Research Award: An exhilarating journey to the DNA damage checkpoint
}

\author{
Lee $\mathrm{Zou}^{1,2^{*}} \& \mathrm{Lei}^{\mathrm{Li}^{*}}$ \\ ${ }^{1}$ Massachusetts General Hospital Cancer Center, Harvard Medical School, Boston, MA 02129, USA; \\ ${ }^{2}$ Department of Pathology, Massachusetts General Hospital, Harvard Medical School, Boston, MA 02114, USA; \\ ${ }^{3}$ The University of Texas M. D. Anderson Cancer Center, Department of Experimental Radiation Oncology, Houston, TX 77030, USA
}

Received December 7, 2015; accepted December 10, 2015; published online December 11, 2015

Citation: Zou L, Li L. (2016). The 2015 Albert Lasker Basic Medical Research Award: An exhilarating journey to the DNA damage checkpoint. Sci China Life Sci 59, 103-105. doi: 10.1007/s11427-015-4984-3

The field of DNA damage response has experienced an extraordinary year in 2015. The Lasker Award in Basic Medical Research recognized Drs. Evelyn Witkin and Stephen J. Elledge for their groundbreaking works in the DNA damage response, followed by the Nobel Chemistry Prize honoring three pioneers in DNA damage repair. These highest awards in science attest to both the impact of DNA damage research and the extraordinary accomplishments of their recipients. The prototype concept of DNA damage response emerged from Evelyn Witkin's study of UV-induced mutagenesis in E. coli in the 1960s. Investigations on DNA damage responses in eukaryotes eventually made it plainly clear how vitally important these cellular mechanisms are in maintaining genetic stability of all living creatures and to human diseases. Here we briefly outline the milestones of DNA damage checkpoint research and the many contributions from Stephen Elledge, which led to his Lasker Award (Figure 1).

\section{INTRODUCTION}

The first discovery of cellular mechanisms countering the mutagenic effect of DNA damage actually preceded the discovery of DNA as the genetic material. In an elegantly told story (Friedberg, 1997), Errol Friedberg described how Albert Kelner stumbled upon photo-reactivation repair of

*Corresponding author (email: zou.lee@mgh.harvard.edu; leili@mdanderson.org)

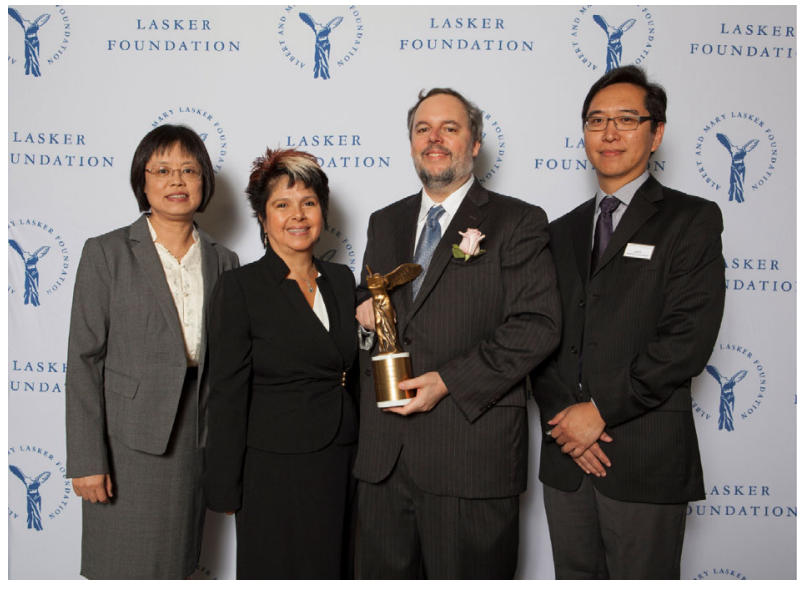

Figure 1 (color online) Stephen Elledge and his former trainees at the Lasker Award ceremony on September 18, 2015, New York. From left to right: Zheng Zhou (Professor, Baylor College of Medicine), Yolanda Sanchez (Associate Professor, Dartmouth College), Steve Elledge (HHMI, Harvard Medical School), and Lee Zou (Professor, Harvard Medical School, Massachusetts General Hospital). Photo credit: Stephen Elledge and the Lasker Foundation.

UV lesions at the Cold Spring Harbor laboratory. In his 1949 paper published in PNAS (Kelner, 1949), Albert Kelner stated:

"While it is premature to do more than to speculate on the mechanism involved in light-induced recovery, the following is suggested as a working hypothesis. Much of the killing effect of ultraviolet-light is due to a light-labile alteration of some constituent in the cell. Exposure to visible light restores this altered constituent to its former state." 
The "constituent in the cell" would not be experimentally established as DNA until 1952 when Hershey and Chase presented the conclusion that DNA is the genetic material of the bacteriophage (Hershey and Chase, 1952).

After DNA was identified as the genetic molecule, the field of DNA damage and mutagenesis has seen its infancy in the early 50s with the term "repair" first used by Salvadore Luria in 1952 (Friedberg, 1997). Over the past 6 decades, the DNA repair and mutagenesis field has evolved from enzymological study of individual DNA repair activities to the complex molecular network of DNA damage response; from the simple UV survival readout of bacterial and phage to cancer etiology and therapeutics. However, it is fair to say that much of what we know about DNA repair benefited in no small ways from early studies in E. coli and yeasts. The spectacular career of Stephen Elledge also followed a similar path of organismal advance with contributions to the field that few, if any, could match.

\section{FROM E. coli TO HUMANS}

The DNA damage response consists of multiple lines of defense against genomic DNA damage, slowing cell cycle progression, modulating DNA repair activities, and utilizing lesion bypass mechanisms to cope with impediments to DNA replication. When Stephen Elledge began his graduate research at MIT with Graham Walker, he was drawn to the DNA damage tolerance and SOS response in E. coli. His work discovered the catalytic subunit of lesion bypass polymerase V (Elledge and Walker, 1983b), which led to the identification of its regulatory subunit UmuD and its damage inducibility as part of the SOS response (Elledge and Walker, 1983a). His Ph.D thesis titled "Identification and characterization of genes involved in mutagenesis in Escherichia coli" has set the stage for his subsequent work on the DNA damage response.

After earning his Ph.D degree in 1983, Steve joined Ron Davis laboratory at Stanford University as a Helen Hay Whitney fellow, combining his exceptional genetics skills and the increasingly powerful budding yeast system. He had a clear objective to begin his postdoc work with - to clone the yeast RecA homolog, so that the eukaryotic counterpart of the SOS pathway would be unraveled. As he recounted recently (Elledge, 2015), an in situ screen of a yeast genomic DNA expression library using $E$. coli RecA antibodies fortuitously identified the small subunit of ribonuclease reductase (RNR2) gene. Even though RNR expression is highly induced upon DNA damage (Elledge and Davis, 1989), the cis-element mediating the inducibility does not resemble that of the E. coli lexA gene. His work, however, defined a new mode of regulating DNA damage response genes in eukaryotic cells.

In 1989, Steve was recruited to the faculty of Baylor College of Medicine, where he continued to study the regulatory mechanism controlling RNR expression in response to DNA damage. In a yeast genetic screen for mutants unable to induce RNR genes, the protein kinase DUN1 has emerged as a key regulator of the DNA damage response. This was a first piece of evidence showing that a protein kinase reacts to DNA damage, revealing the signal transduction pathway governing the DNA damage response (Zhou and Elledge, 1993). Subsequent studies by the Elledge lab has identified the key proteins that mediate damage signaling, revealing a kinase cascade comprised of Mec1, Rad53, and Dun1, and many of their regulators and effectors. By this time, the concept of cell cycle checkpoint had been proposed to explain the delay of cell cycle progression upon DNA damage. Nonetheless, how cells enact such control over the complex cell cycle machinery was by no means perspicacious. In a seminal review in Science, "Cell cycle checkpoints: preventing an identity crisis", Steve formulated his visions of the biochemical nature, signaling mechanisms, and effector engagement of the cell cycle checkpoint (Elledge, 1996). His visions have played a major role in placing the concept of checkpoint in many different biological contexts, greatly enhancing the appreciation of this cellular process. His predictions turned out to be both visionary and accurate. The main framework of the checkpoint has been proven to be the signal transduction cascade he had envisaged, mediated by protein post-translational modifications through phosphorylation and subsequently expanding to many other forms such as ubiquitination and sumoylation.

While his laboratory was busy delineating the DNA damage checkpoint pathways in yeast, Steve began to shift his attention to the DNA damage response in mammalian cells. The yeast model that he built has paved a solid path to the identification of many mammalian DNA checkpoint proteins, such as CHK1 and CHK2 (Sanchez et al., 1996; Sanchez et al., 1997). As more and more mammalian checkpoint proteins emerged, the key question regarding checkpoint activation remained - How does the checkpoint recognize the presence of DNA damage and activate the signaling cascade? Given that DNA damages vary tremendously in chemical and structural forms, it is unlikely that a few checkpoint sensing factors are able to recognize all forms of DNA lesions. This fundamental question was answered when the DNA damage-induced binding of RPA, the predominant single-stranded DNA (ssDNA) binding protein in human cells, and ATRIP, the regulatory partner of the checkpoint initiating kinase ATR, was identified (Zou and Elledge, 2003). By exposing ssDNA, both DNA damage repair intermediates and replication fork stress can trigger checkpoint activation. This paradigm of DNA damage sensing through ssDNA, conserved in both yeast and humans, has revealed a remarkable similarity between the checkpoint response in eukaryotes and the SOS response in bacteria. Steve's work on DNA damage responses from $E$. coli to humans has completed a full circle. 


\section{BEYOND THE CHECKPOINT}

In addition to his groundbreaking work on the DNA damage response, Steve has made many seminal contributions to other research areas. For many years, Steve has been a pioneer in the cell cycle field. He was one of the first to identify the CDK inhibitor p21. He has also played a critical role in the discovery of cullin family ubiquitin ligases. In recent years, Steve has been a leader in combined genomic and genetic studies using human cells, making major discoveries in the DNA damage response, protein degradation, cancer therapy, and HIV infection. Steve's achievements are always accompanied by his exceptional aptitude in technology development. Even as a graduate student, he was already significantly accomplished in devising molecular genetics-based functional screens. The $\lambda \mathrm{ACT}$ cDNA library he designed allowed the yeast two-hybrid system to be deployed as a common protein-protein interaction screening and testing tool in every laboratory (Durfee et al., 1993). As soon as the RNA interference technology emerged, Steve was among the few leaders who constantly revamped the design and efficiency of this powerful platform. He has provided the field with many crucial resources including the atlas of ubiquitinated proteins and ATM/ATR substrates. More recently, his lab invented a viral infection profiling method that detects a person's "virome" with a minute amount of blood sample (Xu et al., 2015). The many conceptual and practical innovations in technology advancement broadened his research impact far beyond the genomic instability field.

The field of DNA damage response has experienced tremendous growth. Its impact on cancer etiology and cancer therapy is becoming increasingly clear. The ever-growing knowledge from the DNA damage field has enabled us to better understand how cancer cells evolve, and also to target them based on their intrinsic DNA damage response deficiencies. Direct and indirect DNA-targeting therapies, from proton particle radiation therapy to the ever-improving platinum drugs, may be applied with more efficacies based on the DNA damage response of the tumor. Mechanistic delineation of the DNA damage response also guides the creation of drugs already in clinics, such as PARP inhibitors, or in clinical trials, such as the ATR, CHK1 and WEE1 inhibitors. These advances in improving human health attest to the impactful research of the DNA damage response collectively, and the extraordinary accomplishments of Stephen Elledge who, with Evelyn Witkin, is the most deserving recipient of the 2015 Albert Lasker award for Basic Medical Research.

Compliance and ethics The author(s) declare that they have no conflict of interest.

Durfee, T., Becherer, K., Chen, P.L., Yeh, S.H., Yang, Y., Kilburn, A.E., Lee, W.H., and Elledge, S.J. (1993). The retinoblastoma protein associates with the protein phosphatase type 1 catalytic subunit. Genes Dev 7, 555-569.

Elledge, S.J. (1996). Cell cycle checkpoints: preventing an identity crisis. Science 274, 1664-1672.

Elledge, S.J. (2015). Accidents and damage control. Cell 162, 1196-1200.

Elledge, S.J., and Davis, R.W. (1989). DNA damage induction of ribonucleotide reductase. Mol Cell Biol 9, 4932-4940.

Elledge, S.J., and Walker, G.C. (1983a). The muc genes of pKM101 are induced by DNA damage. J Bacteriol 155, 1306-1315.

Elledge, S.J., and Walker, G.C. (1983b). Proteins required for ultraviolet light and chemical mutagenesis. Identification of the products of the umuC locus of Escherichia coli. J Mol Biol 164, 175-192.

Friedberg, E.C. (1997). Correcting the blueprint of life: An historical account of the discovery of DNA repair mechanisms (Cold Spring Harbor Laboratory Press).

Hershey, A.D., and Chase, M. (1952). Independent functions of viral protein and nucleic acid in growth of bacteriophage. J Gen Physiol 36, 39-56.

Kelner, A. (1949). Effect of visible light on the recovery of Streptomyces Griseus Conidia from Ultra-violet irradiation injury. Proc Natl Acad Sci USA 35, 73-79.

Sanchez, Y., Desany, B.A., Jones, W.J., Liu, Q., Wang, B., and Elledge, S.J. (1996). Regulation of RAD53 by the ATM-like kinases MEC1 and TEL1 in yeast cell cycle checkpoint pathways. Science 271, 357-360.

Sanchez, Y., Wong, C., Thoma, R.S., Richman, R., Wu, Z., Piwnica-Worms, H., and Elledge, S.J. (1997). Conservation of the Chk1 checkpoint pathway in mammals: Linkage of DNA damage to Cdk regulation through Cdc25. Science 277, 1497-1501.

Xu, G.J., Kula, T., Xu, Q., Li, M.Z., Vernon, S.D., Ndung'u, T., Ruxrungtham, K., Sanchez, J., Brander, C., Chung, R.T., O'Connor, K.C., Walker, B., Larman, H.B., Elledge, S.J. (2015). Viral immunology. Comprehensive serological profiling of human populations using a synthetic human virome. Science 348 , aaa0698.

Zhou, Z., and Elledge, S.J. (1993). DUN1 encodes a protein kinase that controls the DNA damage response in yeast. Cell 75, 1119-1127.

Zou, L., and Elledge, S.J. (2003). Sensing DNA damage through ATRIP recognition of RPA-ssDNA complexes. Science 300, 1542-1548

Open Access This article is distributed under the terms of the Creative Commons Attribution License which permits any use, distribution, and reproduction in any medium, provided the original author(s) and source are credited. 